\title{
Mapping spin structures on the atomic scale
}

[DOI: 10.1051/EPN:2007004]

\author{
Roland Wiesendanger, \\ Institute of Applied Physics, University of Hamburg, D-20355 Hamburg • Germany \\ Email:wiesendanger@physnet.uni-hamburg.de.Web:www.nanoscience.de
}

\begin{abstract}
fundamental understanding of magnetic and spin-dependent phenomena requires the determination of spin structures and spin excitations down to the atomic scale. The direct visualization of atomic-scale spin structures [1-4] has first been accomplished for magnetic metals by combining the atomic resolution capability of Scanning Tunnelling Microscopy (STM) $[5,6]$ with spin sensitivity, based on vacuum tunnelling of spin-polarized electrons [7]. The resulting technique, SpinPolarized Scanning Tunnelling Microscopy (SP-STM), nowadays provides unprecedented insight into collinear and non-collinear spin structures at surfaces of magnetic nanostructures and has already led to the discovery of new types of magnetic order at the nanoscale [8]. More recently, the detection of spin-dependent exchange and correlation forces has allowed a first direct real-space observation of spin structures at surfaces of antiferromagnetic insulators [9]. This new type of scanning probe microscopy, called Magnetic Exchange Force Microscopy (MExFM), provides a powerful new tool to investigate different types of spin-spin interactions based on direct-, super-, or RKKY-type exchange down to the atomic level. By combining SP-STM with inelastic electron tunnelling spectroscopy [10] or by performing MExFM together with high-precision measurements of damping forces [9] localized or confined spin excitations in magnetic systems of reduced dimensions now become experimentally accessible.
\end{abstract}

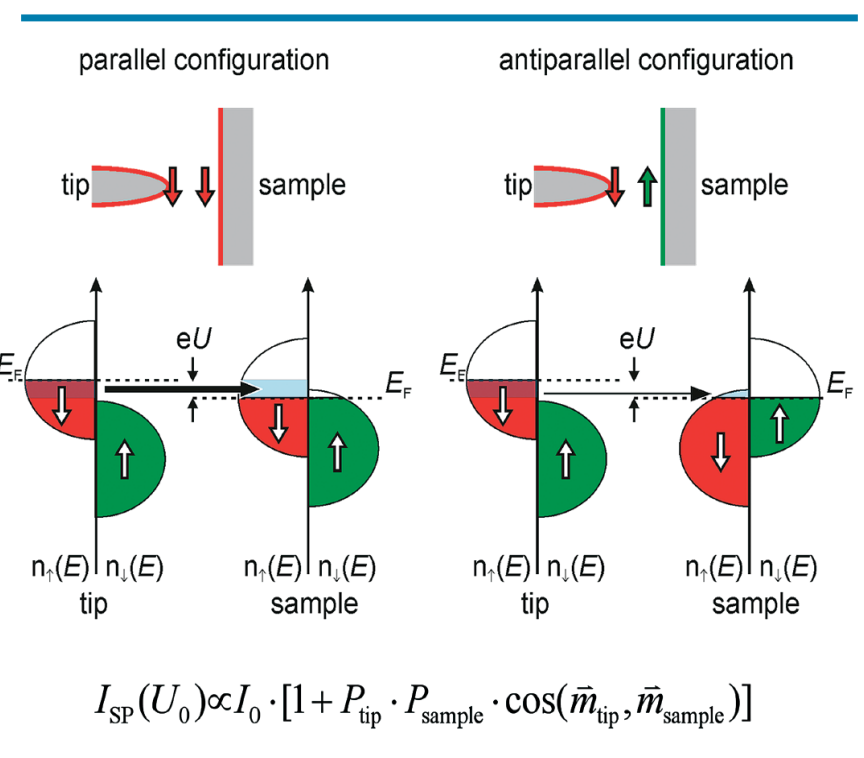

\footnotetext{
$\Delta$ Fig. 1: Principle of Spin-Polarized Scanning Tunnelling Microscopy (SPSTM): the spin-polarized tunnelling current flowing between a magnetic tip and a magnetic sample depends on the relative alignment of the local magnetization of tip and sample as well as on the spin polarization of electronic states of tip and sample contributing to the tunnelling current.
}

\section{Principles of Spin-Polarized Scanning Tunnelling Microscopy (SP-STM) and Spectroscopy (SP-STS)}

The technique of SP-STM is based on vacuum tunnelling of spin-polarized electrons as can be observed in STM-type tunnel junctions involving a magnetic tip and a magnetic sample (Fig. 1). In this case, the tunnelling current does not only depend on the tip-surface separation and the applied bias voltage between tip and sample, but also on the spin polarization of the electronic states near the Fermi level of both electrodes as well as on the relative orientation of the local magnetization of the sample and the magnetic moment at the tip apex. Early experiments based on planar tunnel junctions involving magnetic electrodes have been performed by Jullière in the midseventies [11]. The invention of the STM by G. Binnig and H. Rohrer [5] allowed the replacement of the ill-defined tunnel barrier of planar tunnel junctions by a well defined vacuum barrier and the replacement of one of the planar electrodes by a freely movable and positionable probe tip. Magnetic tips have first been employed in scanning probe microscopy to perform spatially resolved measurements of magnetic dipole forces [12]. The corresponding technique, Magnetic Force Microscopy (MFM), has become a routine method for magnetic domain imaging [13]. However, the spatial resolution of MFM is limited to $10-20 \mathrm{~nm}$ due to the long-range nature of the magnetic dipole forces [6]. In order to improve the spatial resolution of magnetic-sensitive scanning probe microscopy down to the atomic scale, two different approaches have been proposed at the end of the eighties: either to measure spin-dependent tunnelling currents making use of the strong exponential dependence of the tunnelling current on the tip-surface distance in order to combine atomic- with spin-resolution, or to measure spin-dependent exchange and correlation forces at very small distances between a magnetic probe tip and a magnetic sample based on the atomic force microscopy (AFM) technique [14].

The first successful observation of vacuum tunnelling of spin-polarized electrons in an STM experiment was made in 1990 [7] using a highly spin-polarized $\mathrm{CrO}_{2}$ thin film probe tip and an antiferromagnetic $\mathrm{Cr}(001)$ sample in order to exclude a disturbing influence of superimposed magnetic dipole interactions. In these early SP-STM experiments, the constant-current mode of operation was employed where spin-polarized tunnelling current effects showed up as apparent height differences in constant-current STM contours. This mode of operation has turned out to be useful for atomic-scale SP-STM studies of surface spin configurations, as has been shown by resolving the spin structure at surfaces of ferrimagnetic oxides [1], two-dimensional antiferromagnetic metal layers [2-4] and antiferromagnetic nitrides [15]. For nanoscale magnetic domain imaging the spectroscopic mode of SP-STM operation, introduced in 1998 [16], was found to be superior compared to the constant-current mode, particular with respect to a clear separation between 
topographic, electronic, and magnetic contrast effects [17,18]. In the spectroscopic mode of SP-STM the spin-resolved differential tunnelling conductance $\mathrm{dI} / \mathrm{dU}$ is measured with spatial resolution. The bias voltage applied between the magnetic tip and the magnetic sample is chosen in such a way that tunnelling into or out of a highly spin-polarized electronic state leads to a high spin contrast image. The advances which came along with the spectroscopic mode of SP-STM (called SP-STS) operation is nicely demonstrated by experiments revealing the magnetic domain structure of rare-earth metal films, such as Dy(0001) epitaxially grown on W(110) single crystal substrates [19].

\section{Ultra-high resolution magnetic domain imaging by SP-STS}

In Fig. 2 simultaneously recorded topographic and magnetic maps of a Dy(0001) thin film are presented. While the topographic STM image (a) reveals a relatively smooth surface structure with atomically flat terraces together with some surface steps and several dislocations, the SP-STS image (b) clearly shows the magnetic domains with very sharp transitions (magnetic domain walls) between them. Since the magnetic anisotropy is in the plane of the Dy film, an in-plane magnetized thin film probe tip has been used in order to achieve a high spin contrast. Additionally, the applied sample bias voltage has been chosen in such a way that highly spin-polarized electronic states are involved in tunnelling. The observed six different contrast levels in the magnetic SP-STS image result from the six equivalent in-plane directions of the local sample magnetization reflecting the six-fold symmetry of the underlying hexagonal crystal lattice structure of the Dy film. For a given tip magnetization direction (quantization axis) this leads to six different projections (mathematically described by the cosinedependence of the spin-polarized tunnelling current on the angle between the tip and sample magnetization directions, see
Fig. 1) which can clearly be identified in the histogram of the measured values of the spin-resolved $\mathrm{dI} / \mathrm{dU}$ signal across the whole image (see Fig. 2c). The very sharp transitions between the observed magnetic domains result from the relatively high magnetic anisotropy of the Dy film leading to very narrow domain walls of only $2-3 \mathrm{~nm}$ in width. For single atomic layers of Fe on W(110) substrates even atomically sharp domain walls have been revealed by SP-STS [18]. In this case, the magnetic anisotropy of the single atomic layer of Fe on W(110) is much larger than for bulk Fe where domain wall widths are typically on the order of a hundred nanometers.

It should be emphasized that a reliable quantitative determination of magnetic domain wall widths requires the use of magnetic stray field-free tips in scanning probe microscopy. This has always been a severe problem in MFM studies of magnetic domain walls. However, the problem can be solved based on the SP-STM method by introducing antiferromagnetically coated probe tips $[20,21]$ which still exhibit spin-polarized electronic states for tunnelling but are strayfield-free. Therefore, they do not affect the position or the measured width of magnetic domain walls or magnetic vortices [21]. Moreover, it has been demonstrated that even a non-magnetic probe tip may be able to image domain walls since the local electronic structure is modified within the domain wall due to the local change of the magnetization direction and the accompanied change of the spin-orbit coupling between electronic states [22,23].

To summarize this part, SP-STS allows us to enter a new regime of magnetic domain and domain wall observation at sub-nanometer scale spatial resolution which is not accessible by any other magnetic imaging technique up to now.

\section{Spin-resolved electronic structure at the nanoscale}

The real value of the STM technique is not just its high spatial resolution but is also that the combination with tunnelling

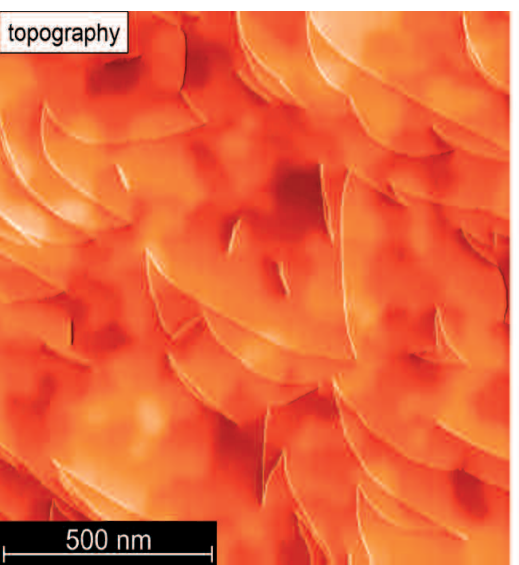

a)

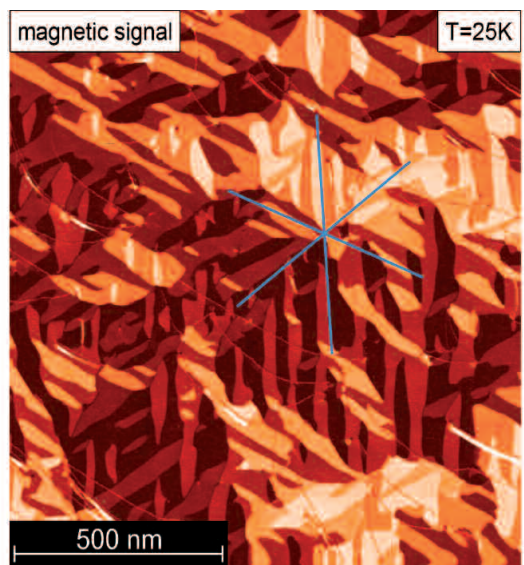

b)

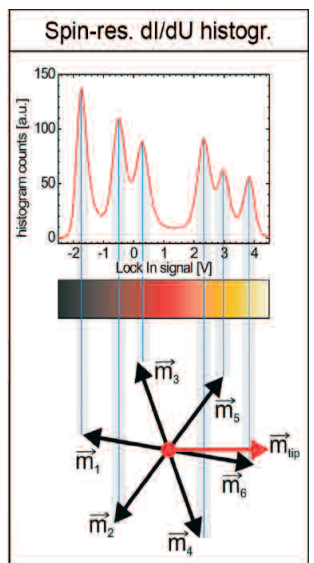

c)
Fig. 2: Application of SP-STS towards magnetic domain imaging with sub-nanoscale spatial resolution: a thin Dy(0001) film grown epitaxially on a W(110) substrate exhibits a domain structure with six different in-plane orientations of the local magnetization. The six different contrast values in the SP-STM image result from the six different projections of the local sample magnetization onto the local magnetization direction of the probe tip (quantization axis). The relatively high magnetic anisotropy of Dy leads to narrow magnetic domain walls of typically 2 - $3 \mathrm{~nm}$ width [22]. 
spectroscopy provides access to the spatial distribution of electronic states down to the atomic level [5]. Most importantly, this type of atomic-resolution spectroscopy can be performed at low energy scales which are relevant to the investigation of quantum phenomena in nanostructures, in addition to providing a non-destructive imaging method. The combined observation of atomic-scale surface topography and spatial distribution of the electronic states by Scanning Tunnelling Spectroscopy (STS) is illustrated in Fig. 3a. Nanoscale Co islands of triangular shape and two atomic layers height have been prepared on a $\mathrm{Cu}(111)$ surface which exhibits a well known Shockley-type surface state. The surface state electrons scatter at the interface of the Co islands with the $\mathrm{Cu}$ substrate and the reflected electron waves interfere with the incoming waves, resulting in a standing wave pattern near the Co island edges. Surface state electrons are also scattered at individual Co atoms giving rise to circular-symmetric (s-type) scattering states characteristic of free-electron behaviour. On top of the topographically flat Co islands electronic modulations are observed which result from the quantum mechanical lateral confinement of the electrons within the triangular shaped Co islands. While conventional STS methods have already widely been used to study such phenomena on many different surfaces, only SP-STS allows us to address questions related to the magnetic state of nanoscale islands [24], the spin-dependence of the electronic confinement states [25], and the spin dependence of the electronic scattering at nanoscale interfaces or at individual adatoms [26]. Fig. 3b shows examples of four different SP-STS maps obtained at four different energies (sample bias voltages), revealing the spatial distribution of the corresponding spin-polarized electronic states within the single-domain Co islands below and above the onset of the $\mathrm{Cu}$ surface state. The particular energies have been selected in such a way that the corresponding spin asymmetry of the tunnelling current and therefore the spin contrast in the SP-STS image is high. Therefore, the magnetic state of the Co islands with a magnetic out-of-plane anisotropy (either with an upward or a downward magnetization direction) clearly shows up as bright or dark contrast. At positive sample bias voltages the electronic modulations due to the $\mathrm{Cu}(111)$ surface state and due to the laterally confined states within the Co islands are nicely visible in addition. By carefully analyzing the dependence of the amplitude of these electronic modulations on the magnetization state of the respective Co islands, the spin character of the electronic confinement states can be deduced [25]. Even the spin character of one-dimensional confinement states at the rim of such nanoscale Co islands could recently be determined [25].

Notice that these SP-STS experiments have been performed at low temperatures $(14 \mathrm{~K})$ where the nanoscale Co islands are in a ferromagnetic state. At higher temperatures thermal fluctuations may lead to a superparamagnetic behaviour which can be studied by time-dependent SP-STS experiments [27].

\section{Discovery of antiferromagnetic order in Fe monolayers by atomic-resolution SP-STM}

Atomic-resolution mapping of surface spin structures has been a dream for a long time which became a reality for electrically conducting materials by the combination of the STM technique with the development of appropriate spin-polarized probes. A first step towards atomic-scale SP-STM studies was made by resolving the different magnitude of the magnetic moments of octahedrally coordinated $\mathrm{Fe}^{3+}$ and $\mathrm{Fe}^{2+}$ sites at the $\mathrm{Fe}_{3} \mathrm{O}_{4}(001)$ surface [1]. A few years later the different orientation of the magnetic moments of chemically identical $\mathrm{Mn}$ atoms within an antiferromagnetic Mn monolayer prepared on a W(110) substrate was directly observed in real space by SP-STM [2]. Another atomic-resolution SP-STM investigation was reported for a $\mathrm{Mn}_{3} \mathrm{~N}_{2}(010)$ surface by Yang et al. [15]. For all these early SP-STM studies revealing the atomic spin configuration, the constant-current mode of operation was employed, as introduced in 1990 [7]. However, atomic resolution can also be obtained in the spectroscopic mode in which the spin-resolved

\footnotetext{
V Fig. 3: Nanoscale Co islands of two atomic layers height grown on a Cu(111) substrate: (a) Perspective-view STS image exhibiting simultaneously the sample topography and the electronic standing wave patterns on the $\mathrm{Cu}(111)$ surface as well as the quantum mechanical electronic confinement states within the $\mathrm{Co}$ islands. (b) Top-view SP-STS images of the Co-islands on Cu(111) obtained at different samples biases corresponding to energies below or above the onset of the $\mathrm{Cu}(111)$ surface state. The sample bias voltages have been chosen in such a way that the energy-dependent spin asymmetry of the tunnel junction and therefore the magnetic contrast in the SP-STS images becomes large. Notice that the spin asymmetry not only varies in magnitude but even changes sign as a function of energy. At zero-crossings of the spin asymmetry curve no magnetic contrast is obtained [27,28].
}

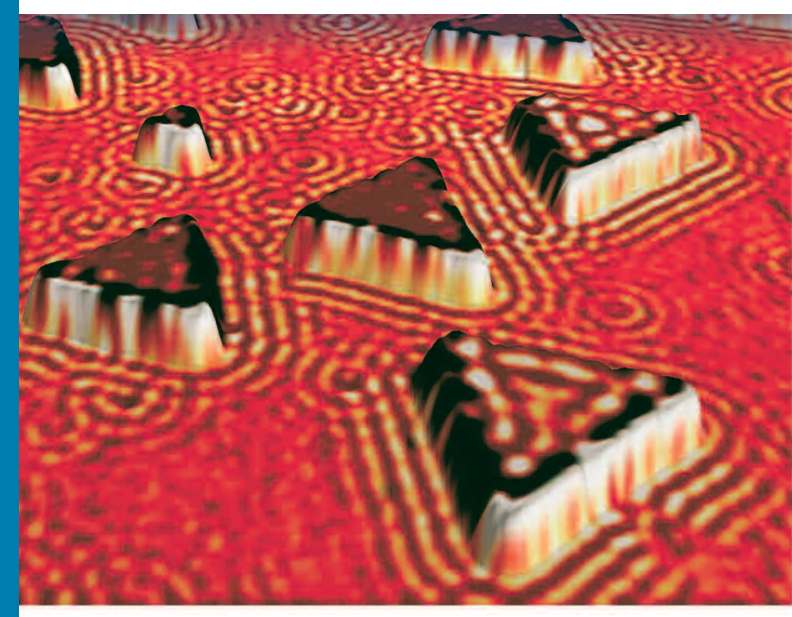

(a)

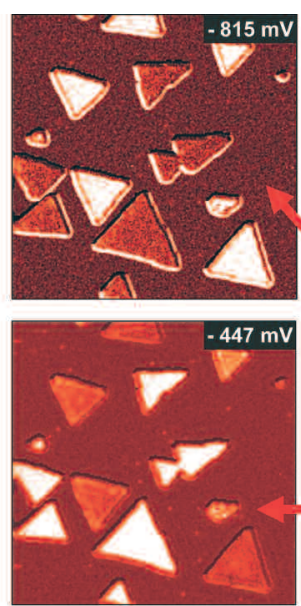

(b)

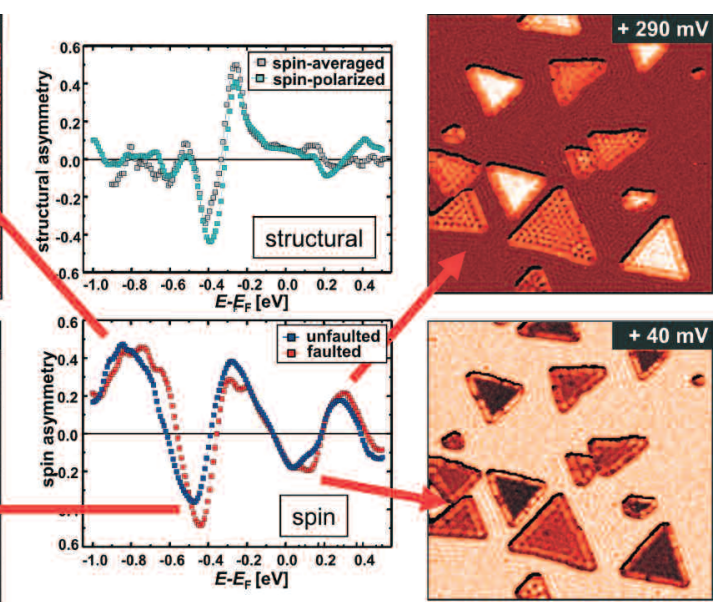

urophysicsnews 


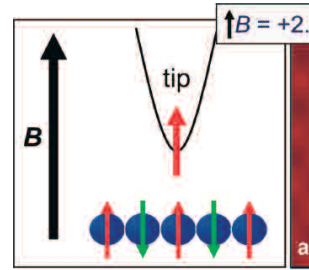

A Fig. 4: Atomic-resolution SP-STM imaging of a recently discovered antiferromagnetic state of a single atomic layer of Fe grown epitaxially on a W(001) substrate: The difference image of
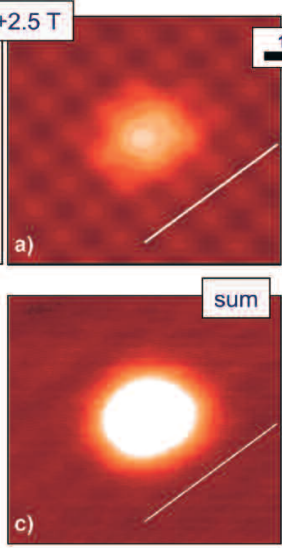

sum
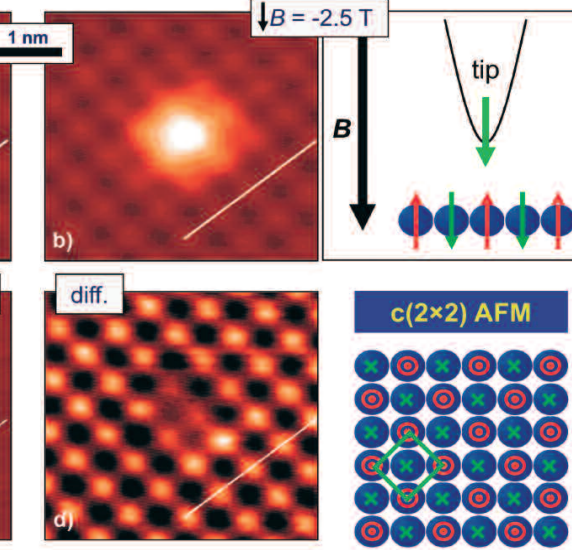

the two data sets (a) and (b) obtained with two oppositely magnetized probe tips results in a magnetic contrast image (d) which reveals for the first time a direct real-space view of Néel-type order on a square lattice [3]. The sum image of (a) and (b) results in a topographic map (c) showing an atomic adsorbate which was used to make the atomic-scale registry between the two images (a) and (b). presented in Fig. 5b. The domain wall width only amounts to $6-8$ atomic rows which has been confirmed by Monte Carlo simulations [4].

The first direct real-space view of non-collinear spin structures as present within the domain wall region of antiferromagnetic layers (Fig. 5b) has triggered several other SP-STM studies of complex spin structures, including a recently discovered mosaic-type spin structure of an Fe monolayer on $\operatorname{Ir}(111)$ with 15 atoms per unit cell, where 7 atoms have their spins pointing in one direction and 8 atoms have their spins pointing in the opposite direction [8]. Another interesting example is given by the recent discovery of cycloidal spin spirals in ultra-thin magnetic layers. differential tunnelling conductance signal $\mathrm{dI} / \mathrm{dU}$ is measured with spatial resolution. An example is presented in Fig. 4 where atomic resolution images of a recently discovered antiferromagnetic ground state of a single atomic layer of $\mathrm{Fe}$ on a $\mathrm{W}(001)$ substrate [3] are presented. At first, a SP-STS image was obtained with a Fe-coated probe tip being magnetized in an external out-of-plane magnetic field of $+2.5 \mathrm{~T}$. A second SP-STS data set was recorded at the same sample location with the same tip but now being magnetized in the opposite direction using an external magnetic field of $-2.5 \mathrm{~T}$. By calculating the difference image of these two data sets, the Néel-type spin order on the square (001) lattice can clearly be revealed whereas the sum image represents the surface topography with an atomic adsorbate which was used to perform the registry between the two images measured with oppositely magnetized probe tips. The experimentally determined out-of-plane magnetic anisotropy of the antiferromagnetic $\mathrm{Fe}$ monolayer on $\mathrm{W}(001)$ has been confirmed by density-functional theory (DFT) calculations taking spin-orbit coupling into account [3].

Since the reproducibility of SP-STM and SP-STS imaging is nowadays at the same level as for conventional STM experiments we can take a series of atomicresolution SP-STM data sets at varying sample locations and put them together in order to reveal the spin ordering in the presence of defects (vacancies or adsorbates) and nanoscale islands (Fig. 5a). Interestingly, the perfect Néel-type spin order is maintained right up to the pointdefect sites and step edges. It has proven to be very difficult to find domain walls in the antiferromagnetic Fe monolayer. Only in rare cases where two extended defects are quite close in space were we able to find and study antiferromagnetic domain walls [4]. A detailed view of such a domain wall with atomic and spin resolution is

\section{Mapping atomic-scale spin structures on insulators by MExFM}

While SP-STM and SP-STS methods have provided unprecedented insight into atomic spin configurations at surfaces, their application is limited to electrically conducting samples such as magnetic metal films or magnetic semiconductors. In order to reveal atomic spin structures at surfaces of insulators and to open up the exciting possibility of studying spin ordering effects with atomic resolution while going through a metalinsulator transition, we have developed the technique of Magnetic Exchange Force Microscopy (MExFM). This technique is based on the detection of short-range spin-dependent exchange and correlation forces at very small tip-sample separations (a few Angströms), in contrast to MFM where the magnetic dipole forces are probed with a ferromagnetic probe tip at a typical tip-to-surface distance of $10-20 \mathrm{~nm}$. An important
₹ Fig. 5: (a) Perspective-view of several atomic-resolution SP-STM images of the antiferromagnetic monolayer of Fe on W(001) put together in order to reveal the perfect antiferromagnetic spin ordering even up to atomic-scale defects (vacancies and adsorbates) and double-layer high Fe islands. Notice the small signal strength originating from the periodic spin lattice in comparison to the signal originating from single adsorbed atoms or single-atom high steps. (b) Atomic-resolution SP-STM image of a domain wall in the antiferromagnetic Fe monolayer. The domain wall width only amounts to 6 - 8 atomic rows [4].

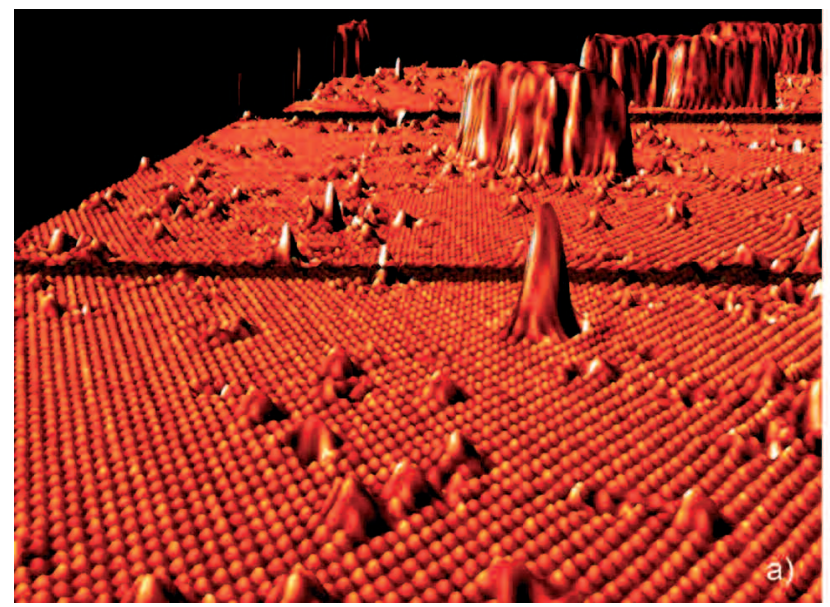

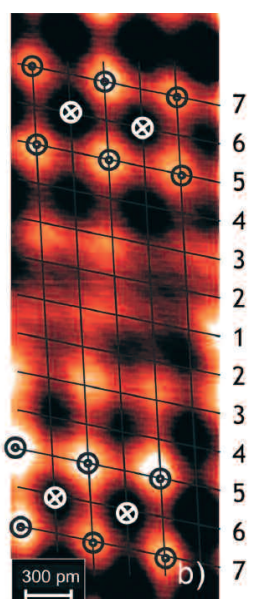


starting point for achieving the challenging goal of atomicresolution spin mapping on surfaces of insulators has been the development of non-contact atomic force microscopy (NCAFM) with true atomic resolution [28]. Nowadays, NC-AFM allows atomically resolved studies of any material system [29], even in the case of curved surface topographies [30]. MExFM combines the possibilities of NC-AFM and atomic-scale spin resolution by making use of an atomically sharp probe tip with a very well defined spin state at its apex. Based on the knowledge gained during the development of SP-STM in preparing such tips we have recently succeeded in resolving the surface spin structure of the antiferromagnetic insulator $\mathrm{NiO}(001)$ [9]. Fig. 6a shows an atomic-resolution topographic NC-AFM image revealing chemical contrast between the oxygen atoms (bright sites) and the $\mathrm{Ni}$ atoms (dark sites) which originates from a different total charge density above $\mathrm{O}$ - and $\mathrm{Ni}$-sites. No contrast is observed between magnetically inequivalent $\mathrm{Ni}$ sites, i.e. $\mathrm{Ni}$ atoms with a different orientation of their magnetic moments. By approaching the out-of-plane magnetized Fecoated tip closer to the surface atoms, the spin-dependent exchange interaction between the rather localized Ni d-states of the sample and the Fe d-states of the tip leads to a different force or force gradient above $\mathrm{Ni}$ atoms with a different orientation of their magnetic moments. As a result, a superperiodicity corresponding to an antiferromagnetically ordered state of the $\mathrm{NiO}(001)$ surface is observed in the MExFM image (Fig. $6 \mathrm{~b})$. Notice that the apparent height difference between the magnetically inequivalent $\mathrm{Ni}$-sites, corresponding to the different magnitude of the spin-dependent quantum-mechanical forces felt by the tip above the different $\mathrm{Ni}$ atoms, only amounts to $1.5 \mathrm{pm}$ as can be deduced from the line section in Fig. 6b. To resolve such tiny signals, the AFM instrument has to be operated at low temperatures in order to reduce the thermal excitations of the AFM cantilever (force sensor).

To summarize, it has become possible to measure spindependent quantum mechanical exchange forces between a single atom at a tip apex and individual surface atoms, leading to a new type of scanning probe microscopy technique which can now resolve atomic spin structures at surfaces of any type of material system, thereby extending the range of applications of SP-STM considerably. Interestingly, it has taken more than a hundred years after the foundations of quantum mechanics have been formulated, to be finally able to measure the spindependent forces between quantum objects directly.

\section{Acknowledgments}

I would like to thank my coworkers and collaborators L. BerbilBautista, K. von Bergmann, S. Blügel, M. Bode, P. Ferriani, S. Heinze, U. Kaiser, S. Krause, A. Kubetzka, A. Lichtenstein, O. Pietzsch, A. Schwarz, and E. Vedmedenko for their contributions. Financial support from the DFG Center of Excellence SFB 668 is gratefully acknowledged.

\section{About the author}

Roland Wiesendanger studied Physics at the University of Basel where he received his Ph.D. in 1987. Since 1989 he has been working towards magnetic imaging with atomic resolution. In 1993 he became full professor (C4) at the University of Hamburg where he initiated several nanoscience centers. He has already received several awards, among them the Karl Heinz Beckurts Prize, the Philip Morris Research Prize, the Gaede Prize, and the Max Auwärter Prize. He is an elected member of the German Academy Leopoldina and the Hamburg Academy of Sciences.

\section{References}

[1] R. Wiesendanger, I.V. Shvets, D. Bürgler, G. Tarrach, H.-J. Güntherodt, J.M.D. Coey, and S. Gräser, Science 255, 583 (1992); R. Wiesendanger, I.V. Shvets, D. Bürgler, G. Tarrach, G. Güntherodt, H.-J. Güntherodt, and J.M.D. Coey, Europhys. Lett. 19, 141 (1992)

[2] S. Heinze, M. Bode, O. Pietzsch, A. Kubetzka, X. Nie, S. Blügel, and R. Wiesendanger, Science 288, 1805 (2000)

[3] A. Kubetzka, P. Ferriani, M. Bode, S. Heinze, G. Bihlmayer, K. von Bergmann, O. Pietzsch, S. Blügel, and R. Wiesendanger, Phys. Rev Lett. 94, 087204 (2005)

[4] M. Bode, E.Y. Vedmedenko, K. von Bergmann, A. Kubetzka, P. Ferriani, S. Heinze, and R. Wiesendanger, Nature Materials 5, 477 (2006)

[5] G. Binnig and H. Rohrer, Rev. Mod. Phys. 59, 615 (1987)

[6] R. Wiesendanger, "Scanning Probe Microscopy and Spectroscopy: Methods and Applications", Cambridge University Press, Cambridge 1994
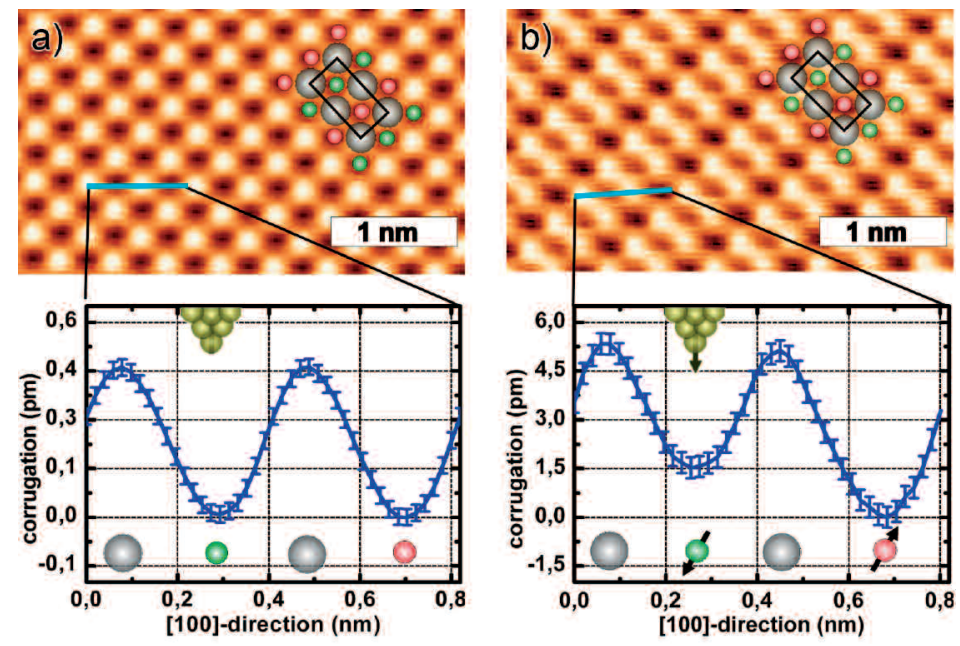

4 Fig. 6: (a) Atomic-resolution image of an antiferromagnetic $\mathrm{NiO}(001)$ surface obtained by non-contact atomic force microscopy (NC-AFM) exhibiting pure chemical contrast. The line section reveals an apparent height difference of 4.5 pm between nickel (dark) and oxygen (bright) sites. Unit cell averaging has been employed in order to improve the signal-to-noise ratio in this data set. (b) Spinresolved image of $\mathrm{NiO}(001)$ with atomic resolution as obtained by Magnetic Exchange Force Microscopy (MExFM) after unit cell averaging. While the oxygen atoms reveal the same contrast throughout the image the nickel atoms with opposite spin orientations now show up with an apparent height difference of $1.5 \mathrm{pm}$. This small height difference in the MExFM image results from the magnetic exchange interaction between the magnetic atom at the tip apex and the magnetic atoms on the sample surface depending on the relative spin alignment. Notice that the spin-dependent exchange and correlation forces on the antiferromagnetic $\mathrm{NiO}$ surface can only be measured at very small tip-surface distances due to the localized nature of the $\mathrm{Ni}$ d-states in the insulating $\mathrm{NiO}$ sample [10]. 
[7] R. Wiesendanger, H.-J. Güntherodt, G. Güntherordt, R.J. Gambino, and R. Ruf, Phys. Rev. Lett. 65, 247 (1990)

[8] K. von Bergmann, S. Heinze, M. Bode, E.Y. Vedmedenko, G. Bihlmayer, S. Blügel, and R. Wiesendanger, Phys. Rev. Lett. 96, 167203 (2006)

[9] U. Kaiser, A. Schwarz, and R. Wiesendanger, Nature (2007), in press

[10] T. Balashov, A.F. Takács, W. Wulfhekel, and J. Kirschner, Phys. Rev. Lett. 97, 187201 (2006)

[11] M. Jullière, Phys. Lett. A 54, 225 (1975)

[12] Y. Martin and K. Wickramsinghe, Appl. Phys. Lett. 50, 1455 (1987); J. J. Saenz, N. Garcia, P. Grütter, E. Meyer, H. Heinzelmann, R. Wiesendanger, L. Rosenthaler, H.R. Hidber, and H.-J. Güntherodt, J. Appl. Phys. 62, 4293 (1987)

[13] A. Schwarz, M. Liebmann, U. Kaiser, R. Wiesendanger, T.W. Noh, and D.W. Kim, Phys. Rev. Lett. 92, 077206 (2004)

[14] G. Binnig, C.F. Quate, and Ch. Gerber, Phys. Rev. Lett. 56, 930 (1986)

[15] H. Yang, A.R. Smith, M. Prikhodko, and W.R.L. Lambrecht, Phys. Rev. Lett. 89, 226101 (2002)

[16] M. Bode, M. Getzlaff, and R. Wiesendanger, Phys. Rev. Lett. 81, 4256 (1998)

[17] M. Kleiber, M. Bode, R. Ravlić, and R. Wiesendanger, Phys. Rev. Lett. 85, 4606 (2000); O. Pietzsch, A. Kubetzka, M. Bode, and R. Wiesendanger, Phys. Rev. Lett. 84, 5212 (2000); O. Pietzsch, A. Kubetzka, M. Bode, and R. Wiesendanger, Science 292, 2053 (2001)

[18] M. Pratzer, H. J. Elmers, M. Bode, O. Pietzsch, A. Kubetzka, and R. Wiesendanger, Phys. Rev. Lett. 87, 127201 (2001)
[19] S. Krause, L. Berbil-Bautista, T. Hänke, F. Vonau, M. Bode, and R. Wiesendanger, Europhys. Lett. 76, 637 (2006)

[20] A. Kubetzka, M. Bode, O. Pietzsch, and R. Wiesendanger, Phys. Rev. Lett. 88, 057201 (2002)

[21] A. Wachowiak, J. Wiebe, M. Bode, O. Pietzsch, M. Morgenstern, and R. Wiesendanger, Science 298, 577 (2002)

[22] M. Bode, S. Heinze, A. Kubetzka, O.Pietzsch, X. Nie, G. Bihlmayer, S. Blügel, and R. Wiesendanger, Phys. Rev. Lett. 89, 237205 (2002)

[23] E. Y. Vedmedenko, A. Kubetzka, K. von Bergmann, O. Pietzsch, M. Bode, J. Kirschner, H. P. Oepen, and R. Wiesendanger, Phys. Rev. Lett. 92, 077207 (2004)

[24] O. Pietzsch, A. Kubetzka, M. Bode, and R. Wiesendanger, Phys. Rev. Lett. 92, 057202 (2004)

[25] O. Pietzsch, S. Okatov, A. Kubetzka, M. Bode, S. Heinze, A. Lichtenstein, and R. Wiesendanger, Phys. Rev. Lett. 96, 237203 (2006)

[26] K. von Bergmann, M. Bode, A. Kubetzka, M. Heide, S. Blügel, and R. Wiesendanger, Phys. Rev. Lett. 92, 046801 (2004)

[27] M. Bode, O. Pietzsch, A. Kubetzka, and R. Wiesendanger, Phys. Rev. Lett. 92, 067201 (2004)

[28] F. J. Giessibl, Science 267, 68 (1995)

[29] S. Morita, R. Wiesendanger, and E. Meyer (eds.), "Non-contact Atomic Force Microscopy", Springer (2002)

[30] M. Ashino, A. Schwarz, T. Behnke, and R. Wiesendanger, Phys. Rev. Lett. 93, 136101 (2004) 